\title{
Beyond the tip of the iceberg; a new view of the diversity of sulfite- and sulfate-reducing microorganisms
}

\author{
Adrien Vigneron $^{1,2} \cdot$ Perrine Cruaud $^{3} \cdot$ Eric Alsop $^{2,4} \cdot$ Julia R. de Rezende $\mathbb{C}^{5} \cdot$ Ian M. Head ${ }^{1} \cdot$ Nicolas Tsesmetzis $\mathbb{D}^{1,2}$
}

Received: 15 November 2017 / Revised: 9 February 2018 / Accepted: 9 March 2018 / Published online: 28 May 2018

(c) International Society for Microbial Ecology 2018

\begin{abstract}
Sulfite-reducing and sulfate-reducing microorganisms (SRM) play important roles in anoxic environments, linking the sulfur and carbon cycles. With climate warming, the distribution of anoxic habitats conductive to dissimilatory SRM is expanding. Consequently, we hypothesize that novel SRM are likely to emerge from the rare biosphere triggered by environmental changes. Using the dsrB gene as a molecular marker of sulfite-reducers and sulfate-reducers, we analyzed the diversity, community composition, and abundance of SRM in 200 samples representing 14 different ecosystems, including marine and freshwater environments, oil reservoirs, and engineered infrastructure. Up to 167,397 species-level OTUs affiliated with 47 different families were identified. Up to $96 \%$ of these can be considered as "rare biosphere SRM". One third of the dsrB genes identified belonged to uncharacterized lineages. The $d s r B$ sequences exhibited a strong pattern of selection in different ecosystems. These results expand our knowledge of the biodiversity and distribution of SRM, with implications for carbon and sulfur cycling in anoxic ecosystems.
\end{abstract}

\section{Introduction}

Sulfite-reducing and sulfate-reducing microorganisms (SRM) are widespread in anoxic environments, such as marine sediments, hydrothermal vents, oil reservoirs, marine, and freshwaters, where they play significant roles in the biogeochemical sulfur cycle [1, 2]. In marine sediments, sulfate reduction activity can potentially oxidize up to $29 \%$ of the organic carbon pool [3]. Therefore, SRM are major players in the carbon cycle of anoxic environments, degrading, directly or indirectly through syntrophic

Electronic supplementary material The online version of this article (https://doi.org/10.1038/s41396-018-0155-4) contains supplementary material, which is available to authorized users.

Ian M. Head

ian.head@newcastle.ac.uk

1 School of Civil Engineering and Geosciences, Newcastle University, Newcastle Upon Tyne, UK

2 Shell International Exploration and Production Inc., Houston, TX, USA

3 INRA, UMR1062 CBGP, F-34988 Montferrier-sur-Lez, France

4 DOE Joint Genome Institute, Walnut Creek, CA, USA

5 Lyell Centre, Heriot Watt University, Edinburgh, UK associations, a broad range of complex substrates, such as carbohydrates [4] or aromatic compounds [5]. SRM have an important impact on natural and engineered environments mainly through their production of hydrogen sulfide, which is both toxic and corrosive and can modify bioavailability of other chemical elements [1]. With climate warming, enhancing rates of oxygen respiration and eutrophication leading to increased organic carbon deposition [6], distribution of anoxic environments with various environmental settings (different carbon sources and interactions with other microorganisms, presence of trace elements) conducive to growth of SRM is projected to increase significantly [7]. Consequently, we hypothesize that sulfate reducers are likely to emerge from the rare biosphere triggered by environmental changes and the spread of unusual anaerobic habitats, with important consequences for ecosystem health [8]. High-throughput sequencing and quantitative PCR analysis of dissimilatory sulfite reductase $d s r B$ genes from an unrivaled collection of 200 environmental samples, representing 14 different ecosystems, has allowed us to revise our knowledge of the global biodiversity of sulfitereducing and sulfate-reducing microorganisms, and identify novel rare SRM lineages that may potentially become dominant organisms in new environments emerging with environmental changes. 
a)

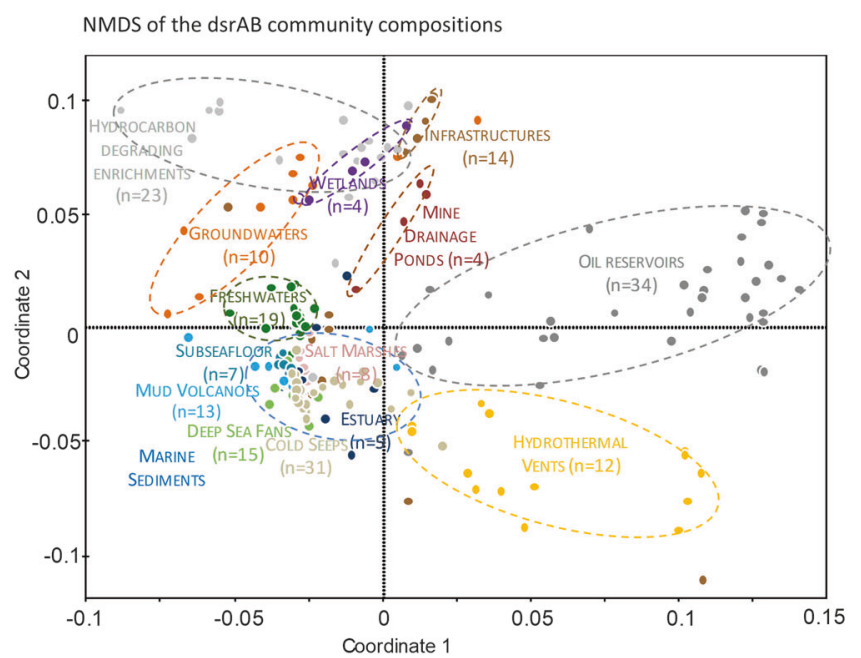

Fig. 1 a Non-metric multidimensional scaling (NMDS) of the $d s r B$ community composition. b Distribution of the 19 most abundant $d s r B$ bearing families in the different ecosystems. Brown, infrastructures; Dark red, mine drainage ponds; purple, wetlands; dark green,

\section{Results and discussion}

In this study, the abundance of sulfite-reducers and sulfatereducers and the composition of the SRM community were investigated using DSR1728f/rDSR4R primer mixes (Supplementary Table 1), targeting the dissimilatory sulfite reductase genes $d s r B$, involved in the last step of the energy producing dissimilatory sulfate reduction pathway and present in all known sulfate-reducing lineages [9-11]. A total of $1.98 \times 10^{7} d s r B$ amplicon sequences were produced from 200 different environmental samples with an average of $1.2 \pm 0.9 \times 10^{5} d s r B$ sequences per sample. After quality filtering (Supplementary material), 167,397 different species-level OTUs (90\% identity cut-off as recommended by Pelikan and coauthors [12]) were identified, increasing substantially previous estimates of potential sulfatereducing microbial diversity that proposed a minimum of 779 different species (OTU level at 90\% identity) [10]. Although this analysis includes a number of microorganisms that carry and express $d s r B$ genes, but do not reduce sulfate, such as Pelotomaculum species [13], Desulfurivibrio alkaliphilus [14]; some members of the Desulfobulbaceae family [15], this clearly, indicates that potential SRM diversity has been considerably underestimated by previous assessments $[10,16]$. In addition, this analysis also includes sulfide oxidizers with oxidative-type DsrAB genes that operate in the reverse direction (labeled as Ox. in Supplementary Material). However, from the total dataset these were represented by only 1885 OTUs $(1.1 \%$ of the OTUs) with an average of $1 \%$ of such sequences per sample. b)

Distribution of the 19 more abundant dsrAB-bearing families in the different ecosystems

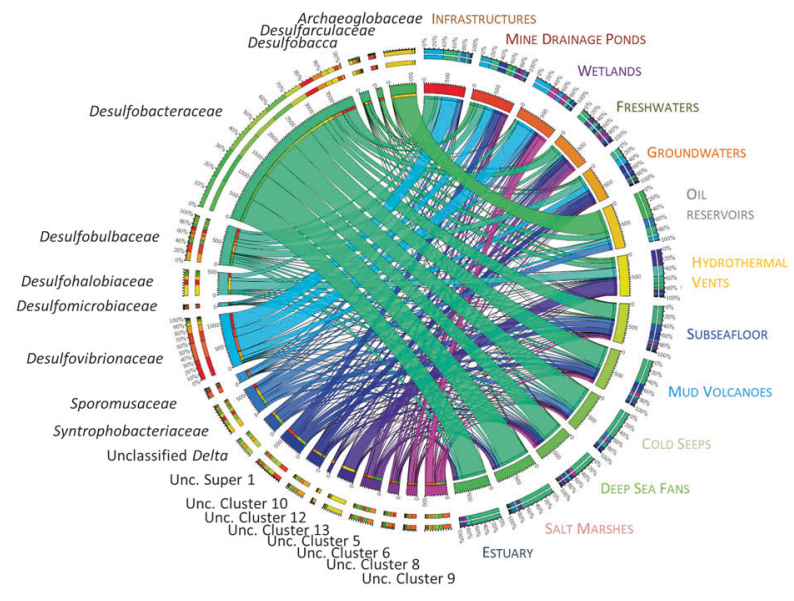

freshwaters; orange, groundwater; grey, oil reservoirs; yellow, hydrothermal vents; dark blue, subseafloor; blue, mud volcanoes; light green, cold seeps; green, deep sea fans; salmon, salt marshes; dark blue, estuary

Considering 240 cultivated species of sulfate reducers, this result also indicates that $<0.2 \%$ of the SRM have been cultivated. Analyses of the distribution of these OTUs in the dataset indicates that rare dsrB OTUs $(<0.1 \%$ in all samples) represented $96.7 \%$ of the OTUs (Supplementary Table 3). This, coupled with the use of low coverage primers, might explain why sulfate reducer diversity has been underestimated previously, using low throughput analyses (e.g., Sanger sequencing of cloned dsrAB genes) [17]. However, these rare sulfite-reducers and sulfate-reducers might represent an important "seed bank" that can have a significant environmental role when triggered by environmental changes [17-19]. The rare SRM biosphere might also include spore-forming sulfate reducers that were previously undetectable by Sanger sequencing without modification of environmental conditions that would lead to germination of their dormant spores [20].

Desulfobacteraceae and Desulfovibrionaceae were the most frequently detected families followed by Desulfohalobiaceae, Desulfobulbaceae, Syntrophobacteraceae, Archaeaoglobaceae, the uncultured cluster 9 of the Environmental supercluster 1 and the uncultured cluster 5 of the Firmicutes supercluster (Fig. 1b, Supplementary Figure 2; [10]). In addition, with the exception of oil reservoirs and corrosive biofilms growing on engineered infrastructure, our results also indicated that $28 \pm 12 \%$ of the detected SRM were affiliated with uncharacterized groups without cultured representatives. Despite the extensive diversity uncovered by deep sequencing, no species-level OTU nor sulfate reducer family was detected as ubiquitous in all environments. Furthermore, community composition among the 
b) dsrAB relative abundance vs richness diversity index
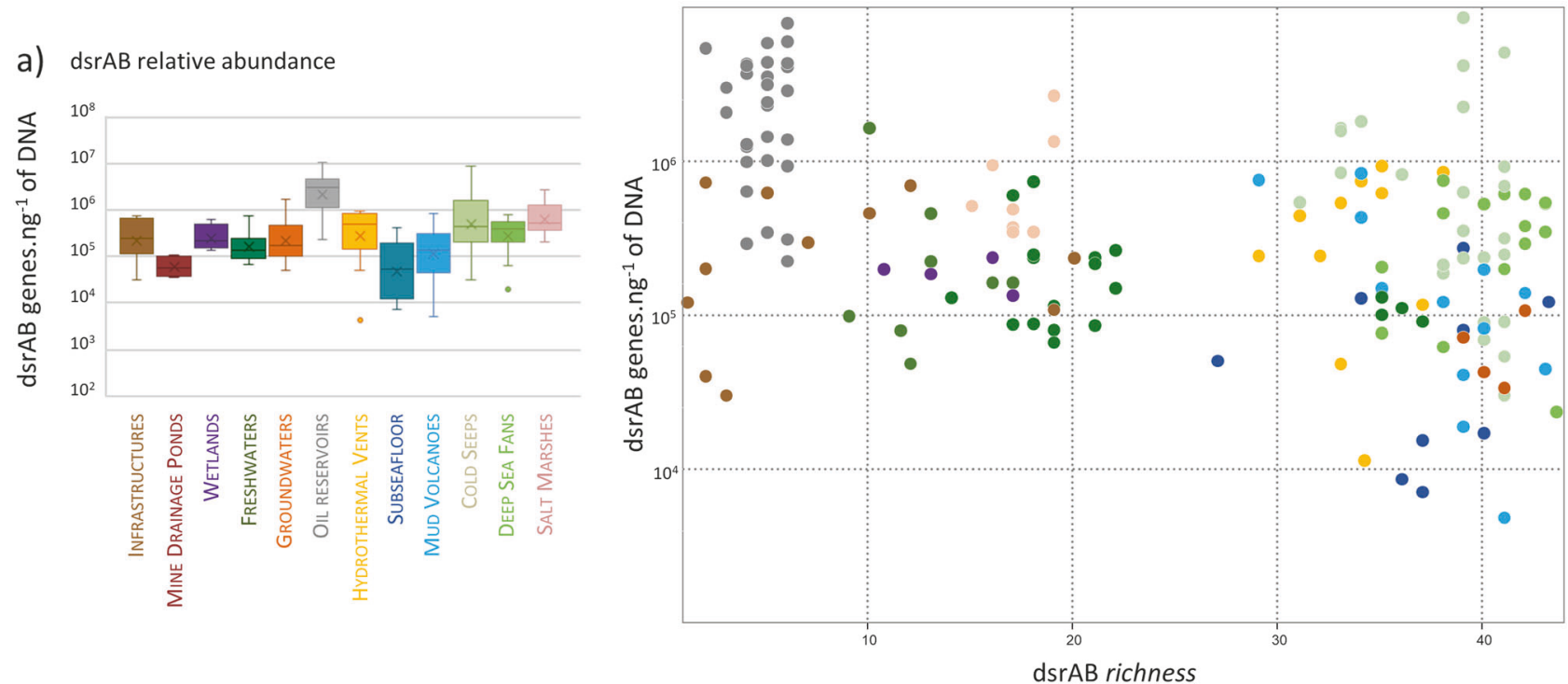

Fig. 2 a DsrB gene abundance (copy per ng of gDNA) in the different sampled environments. Boxes were drawn using 25 and $75 \%$ quartiles, $x$ represents the mean, horizontal line the median, whiskers the variability outside quartiles, and points outside whiskers are outliers. b Relationship between dsrB gene abundance and estimated richness at the family level. Each dot represents. sample. Color of the samples corresponds to the caption in a. Brown, infrastructures; Dark red, mine drainage ponds; purple, wetlands; dark green, freshwaters; orange, groundwater; grey, oil reservoirs; yellow, hydrothermal vents; dark blue, subseafloor; blue, mud volcanoes; light green, cold seeps; green, deep sea fans; salmon, salt marshes. High gene abundance coupled to low richness, as detected in oil reservoirs (grey dots), suggests. strong selective pressure and specialized microorganisms

reducers in marine sediments potentially could be influenced by the availability of utilizable organic carbon [4] and decreases in abundance as labile carbon pools decline. Since climate warming might be associated with increased organic matter deposition, abundance of sulfate-reducing microbes is likely to increase accordingly. The proportion of uncharacterized SRM lineages, as well as members of the Syntrophobacteraceae increased in subseafloor organic poor sediments, suggesting that quality and/or quantity of the labile organic matter might also play a role in shaping the SRM community composition.

Q-PCR results indicated that the abundance of SRM in habitats that will likely expand in the future due to environmental change (e.g. urban freshwater ecosystems, anoxic aquifers, flooded soils, and wetlands) was comparable to their counterparts in marine environments (Fig. 2a). However, a lower richness was observed $(<25 \mathrm{dsrB}$ families) with members of the Desulfobulbaceae and Desulfovibrionaceae, as well as organisms belonging to the uncultured clusters 9 and 10, potentially related to peatland sulfate-reducing Acidobacteria [21]. Although these bacteria might have alternative metabolic capabilities (syntrophic or fermentative lifestyles, nitrate reduction, microaerophilia or sulfide oxidation for some members of the Desulfobulbaceae [15]), these results indicate that, if sulfate concentrations can support their metabolism, unknown lineages of SRM could become important 
components of microbial communities in these expanding environments, potentially leading to substantial release of toxic and corrosive hydrogen sulfide gas.

Acknowledgements Authors thank Anne Godfroy, Laurent Toffin, Ana Suarez-Suarez, Tetyana Korin for providing samples. This work was supported by the METAMORPHE-2 project funded by Shell Global Solutions, the UK Natural Environment Research Councilfunded OILSPORE project (NE/J024325/1) the UK Natural Environment Research Council industrial CASE studentship (NE/J024325/1), and the U.S. Department of Energy Joint Genome Institute (DE-AC0205CH11231).

Funding This work was supported by Shell Global Solutions International BV.

\section{Compliance with ethical standards}

Conflict of interest The authors declare that they have no conflict of interest.

\section{References}

1. Muyzer G, Stams AJM. The ecology and biotechnology of sulphate-reducing bacteria. Nat Rev Micro. 2008;6:441-54.

2. Holmer M, Storkholm P. Sulphate reduction and sulphur cycling in lake sediments: a review. Freshw Biol. 2001;46:431-51.

3. Bowles MW, Mogollón JM, Kasten S, Zabel M, Hinrichs K-U. Global rates of marine sulfate reduction and implications for subsea-floor metabolic activities. Science. 2014;344:889.

4. Rabus R, Venceslau SS, Wöhlbrand L, Voordouw G, Wall JD, Pereira IAC. A post-genomic view of the ecophysiology, catabolism and biotechnological relevance of sulphate-reducing prokaryotes. In: Robert K. Poole, (eds). Advances in Microbial Physiology. Academic Press; 2015; Vol. 66: p. 55-321.

5. Musat F, Galushko A, Jacob J, Widdel F, Kube M, Reinhardt R, et al. Anaerobic degradation of naphthalene and 2methylnaphthalene by strains of marine sulfate-reducing bacteria. Environ Microbiol. 2009;11:209-19.

6. Hoegh-Guldberg O, Bruno JF. The impact of climate change on the world's marine ecosystems. Science. 2010;328:1523.

7. Harley CDG, Randall Hughes A, Hultgren KM, Miner BG, Sorte CJB, Thornber CS, et al. The impacts of climate change in coastal marine systems. Ecol Lett. 2006;9:228-41.

8. Kump LR, Pavlov A, Arthur MA. Massive release of hydrogen sulfide to the surface ocean and atmosphere during intervals of oceanic anoxia. Geology. 2005;33:397-400.

9. Loy A, Duller S, Wagner M. Evolution and ecology of microbes dissimilating sulfur compounds: insights from siroheme sulfite reductases. In: Dahl C, Friedrich CG, (eds). Microbial Sulfur Metabolism. Berlin, Heidelberg: Springer Berlin Heidelberg; 2008. p. 46-59.

10. Muller AL, Kjeldsen KU, Rattei T, Pester M, Loy A. Phylogenetic and environmental diversity of DsrAB-type dissimilatory (bi)sulfite reductases. ISME J. 2015;9:1152-65.

11. Wagner M, Loy A, Klein M, Lee N, Ramsing NB, Stahl DA, et al. (2005). Functional marker genes for identification of sulfatereducing prokaryotes. In: Leadbetter, J.R. (ed)., Methods in Enzymology. Academic Press; 2005; Vol. 397: p. 469-89.

12. Pelikan C, Herbold CW, Hausmann B, Müller AL, Pester M, Loy A. Diversity analysis of sulfite- and sulfate-reducing microorganisms by multiplex dsrA and dsrB amplicon sequencing using new primers and mock community-optimized bioinformatics. Environ Microbiol. 2016;18:2994-3009.

13. Imachi H, Sekiguchi Y, Kamagata Y, Loy A, Qiu Y-L, Hugenholtz $\mathrm{P}$, et al. Non-sulfate-reducing, syntrophic bacteria affiliated with Desulfotomaculum cluster I are widely distributed in methanogenic environments. Appl Environ Microbiol. 2006;72:2080-91.

14. Thorup C, Schramm A, Findlay AJ, Finster KW, Schreiber. (2017). Disguised as a sulfate reducer: growth of the deltaproteobacterium Desulfurivibrio alkaliphilus by sulfide oxidation with nitrate. mBio. 2017. https://doi.org/10.1128/mBio.00671-17.

15. Trojan D, Schreiber L, Bjerg JT, Bøggild A, Yang T, Kjeldsen $\mathrm{KU}$, et al. A taxonomic framework for cable bacteria and proposal of the candidate genera Electrothrix and Electronema. Syst Appl Microbiol. 2016;39:297-306.

16. Colin Y, Goñi-Urriza M, Caumette P, Guyoneaud R. Combination of high throughput cultivation and $d s r$ sequencing for assessment of sulfate-reducing bacteria diversity in sediments. FEMS Microbiol Ecol. 2013;83:26.

17. Hausmann B, Knorr KH, Schreck K, Tringe SG, Glavina del Rio $\mathrm{T}$, Loy A, et al. Consortia of low-abundance bacteria drive sulfate reduction-dependent degradation of fermentation products in peat soil microcosms. ISME J. 2016;10, 2365-2375. https://doi.org/10. 1038/ismej.2016.42.

18. Pester M, Bittner N, Deevong P, Wagner M, Loy A. A 'rare biosphere' microorganism contributes to sulfate reduction in. peatland. ISME J. 2010;4:1591-602.

19. Kalenitchenko D, Le Bris N, Peru E, Galand PE (2018). Ultra-rare marine microbes contribute to key sulfur related ecosystem functions. Mol Ecol 2018;27:1494-1504

20. de Rezende JR, Kjeldsen KU, Hubert CRJ, Finster K, Loy A, Jorgensen BB. Dispersal of thermophilic Desulfotomaculum endospores into Baltic sea sediments over thousands of years. ISME J. 2013;7:72-84.

21. Hausmann B, Pelikan C, Herbold CW, Koestlbacher S, Albertsen $\mathrm{M}$, Eichorst SA, et al. Peatland Acidobacteria with a dissimilatory sulfur metabolism. ISME J 2018; https://doi.org/10.1038/s41396018-0077-1 